\title{
The UP-Philippine General Hospital Acute Coronary Events at the Emergency Room Registry (UP PGH-ACER)
}

\author{
Jean D. Alcover, Ariel D. Valones, Felix Eduardo R. Punzalan and Eugene B. Reyes \\ Section of Cardiology, Department of Medicine, College of Medicine and Philippine General Hospital, University of the Philippines Manila
}

\begin{abstract}
Objectives. This study pilots the creation of a clinical registry for all patients admitted for Acute Coronary Syndromes (ACS) at the Philippine General Hospital (PGH) Emergency Room (ER).

Methods. This is a single-institution prospective observational study including all patients $>18$ years old admitted from September to October 2011 with suspected acute coronary syndromes. Abstraction of chart information using a data collection form was done. Patient's clinical data, medications received, and quality of care indicators were noted. The primary study outcome measure is the completeness of chart data, quality indicators such as door-to-ECG time and door-to-needle time, and the clinical profile of patients with ACS.
\end{abstract}

Results. Thirty patients were included, with equal numbers of males and females, and a mean age of 59 years old. There were equal numbers of patients with STEMI (43\%) and NSTEMI (43\%). The most common risk factor was hypertension (72.4\%) followed by smoking (34.48\%). Around $20 \%$ are diabetics, and $31 \%$ have known lipid abnormalities or are on lipid-lowering agents. Around one third of the patients have had previous admissions for acute coronary events. Only two patients had angiographically confirmed CAD. The majority of patients assessed to have acute coronary syndromes (26 patients) were Killip Class I. Only one was in cardiogenic shock upon admission. Temporal information such as time of first physician contact and time of drug administration was not available in more than half of the cases. While there is a high rate of physician compliance to guideline recommended therapies, temporal quality indicators such as door-to-ECG time (2 hours) and door-to-needle time (3.5 hours) remain substandard. The rest of the patients presented beyond 12 hours of chest pain onset and were already chestpain free. Among those who were eligible for thrombolysis, $67 \%$ received thrombolytic therapy with streptokinase. No patient

Paper presented at the UP PGH Department of Medicine Research Forum, 2011.

Poster presented at the 41st Annual Convention of the Philippine Heart Association and Philippine College of Cardiology, May 2010,

EDSA Shangri-La Hotel, Mandaluyong City.

Corresponding author: Ariel D. Valones MD

Section of Cardiology

Department of Medicine

Philippine General Hospital

University of the Philippines Manila

Taft Avenue, Ermita, Manila 1000 Philippines

Telephone: +632 5548400 local 3670

Email: ariel_valones@yahoo.com was given the thrombolytic agent within 30 minutes upon admission to the emergency department. No patient underwent cardiac catheterization as the primary means of revascularization. Hence, door-to-balloon time could not be determined.

Conclusion. A standardized ACS pathway for adequate documentation of information is necessary for a complete and effective clinical registry for ACS must be set in place. Establishment of an efficient clinical registry must be a joint effort of all services involved in the care for these patients. Proper documentation in clinical charts of patients admitted at the PGH-ER needs to be improved. The quality indicators such as door-to-ECG and door-to-needle time were remarkably above the guideline recommended targets. The compliance for class 1 medications among patients admitted for ACS during the time of the study was optimal.

Key Words: Acute Coronary Syndrome, emergency room, compliance to guidelines, ACS pathway, clinical profile, quality Indicators

\section{Introduction}

Coronary artery disease is a major public health problem all over the world. In the Philippines, statistics from the Department of Health in 2005 show that cardiovascular diseases ranked among the top ten leading causes of morbidity and diseases of the heart and the vascular system are the top two causes of mortality in the country. ${ }^{1}$ Among the more debilitating and economically devastating manifestations of coronary artery disease are acute coronary syndromes.

Acute coronary syndromes (ACS) comprise a spectrum of clinical conditions ranging from unstable angina to nonST-elevation myocardial infarction (NSTEMI) to STelevation myocardial infarction (STEMI). Patients with ACS may present to the emergency room with chest discomfort or angina pectoris. Adjectives frequently used to describe this distress include "viselike," "constricting," "suffocating," "crushing," "heavy," and "squeezing." Other patients may present with "angina equivalents." Anginal "equivalents" (i.e., symptoms of myocardial ischemia other than angina) such as dyspnea, faintness, fatigue, and eructations are common, particularly in the diabetic and elderly groups. ${ }^{2}$

The Philippine Heart Association (PHA) Council on Coronary Artery Disease has recently released the Philippine 
Guidelines on the Management of Coronary Artery Disease. While these guidelines relied heavily on previously published European and American guidelines, its main objective was to improve the quality of care of Filipinos with coronary artery disease (CAD) by defining a local set of standards upon which future recommendations and improvements in health care delivery can be based. ${ }^{3}$ Despite this move, however, there is little available data that describes the contemporary patterns of care and outcome trends among Filipinos admitted for acute coronary syndromes. Data on guideline adherence and performance in terms of generally accepted quality of care measures is likewise lacking.

One of the tools most often used for public health monitoring and quality of care improvement is the disease registry. A disease registry is a database of identifiable persons containing a clearly defined set of health and demographic data collected for a specific public health purpose. The registry collects information that can be used for capturing, managing, and organizing specific information for a population of patients. Disease registries are either clinical-based or population-based. A clinicalbased disease registry contains data on patients with a specific type of disease diagnosed and treated at a practice. ${ }^{4}$ Clinical registries are used for tracking patients' disease presentation, characteristics, risk factors, events, and outcomes. These are being used to record clinical characteristics of patients and all their diseases for the purpose of improving quality of care. They also provide an important opportunity to evaluate current guideline practice. $^{5}$

In 1999, the Global Registry of Acute Coronary Events (GRACE), an international database designed to track the outcomes of patients with coronary syndromes was started. To date, it has included more than 100,000 patients from 247 hospitals from 30 countries all over the world. It has already published a considerable amount of manuscripts, many of which have been instrumental in the modification of clinical practice guidelines that greatly impact the quality of care for ACS. 6 ,7 Moreover, the National Registry of Myocardial Infarction (NRMI) database, which included more than two million ACS patients from the United States since 1990, has identified varying practice patterns and degrees of guideline adherence in real-world clinical practice. Data from this registry shows that adherence to the American Heart Association/American College of Cardiology (AHA/ACC) practice guidelines has improved care for ACS patients and was associated with significant reductions in in-hospital mortality rates. It also underscored the importance of intensifying efforts in identifying gaps in overall care as well as constant quality improvement efforts. ${ }^{8}$

While no such registries exist at the Philippine General Hospital, a prospective study in patients with acute coronary syndrome admitted from October 2006 to September 2007 revealed that compliance to both Class I diagnostic examinations and medications prescribed by the American Heart Association Guidelines was optimal. The use of Class I medications such as aspirin, beta-blockers, angiotensinconverting-enzyme (ACE) inhibitors, and heparin exceeded $90 \%$. This study also reported that among the 54 patients with ST-elevation MI, around $30 \%$ failed to receive reperfusion therapy despite their eligibility. ${ }^{9}$ This figure was similar to United States data, which reported that $28 \%$ of eligible patients fail to receive reperfusion therapy despite the availability of this regimen. The PGH study, however, did not report the timing of the delivery of reperfusion strategies. This information, which includes door-to-needle or door-to-balloon times, was shown by previous studies to greatly affect patient mortality, thus, making it a reliable marker for the quality of health services delivery.

For significant quality of care improvement measures to be set in place, there is a need to describe the current pattern of care in the local setting and to identify key areas of improvement. Because critical aspects of ACS care occur in the emergency room setting, the authors decided to focus on ER management for this registry and provide a framework upon which subsequent registries with wider scopes can be based.

\section{Objectives}

\section{Primary Objective}

To develop a clinical registry for all patients admitted for suspected acute coronary syndromes at the UP-PGH Emergency Room.

\section{Secondary Objectives}

Specifically, the study aims:

1. To describe the clinical profile of ACS patients admitted at the ER.

2. To describe the treatment intervals from ER admission to administration of emergency medications until final patient disposition.

3. To formulate recommendations for improvement in data gathering for a disease registry.

\section{Study Design \\ Prospective observational study}

\section{Methods}

All patients $>19$ years old who consulted at the PGH Emergency Room from September to November 2011 with acute coronary syndrome as a presumptive diagnosis and were alive upon presentation were included. Patients with ACS precipitated or accompanied by significant comorbidities such as trauma or surgery were excluded.

A data collection form (Appendix 1) was used. Data abstracted from the data collection forms was entered into 
an electronic database. All information from specific patients were anonymized and de-identified and was kept strictly confidential.

The patients' clinical profiles, results of diagnostic examinations, medications given and their time of administration were noted. Quality of care was evaluated using indicators based on the 2009 Philippine Heart Association Guidelines on the Management of Coronary Artery Disease from admission up to the time of discharge from the emergency department. (Appendix 2). Eligibility criteria for specific therapies as to aspirin/clopidogrel, ACEI, beta blockers, statins and fibrinolytics (Appendix 3).

Approval from the Department of Medicine's Technical Review Board and the PGH Institutional Review Board was obtained prior to the start of the study. A written approval was also obtained from the Department of Emergency Medicine.

\section{Statistical Analysis}

Descriptive statistics using Excel version 2007 was used to analyze the data. The data was presented in a frequency table in percentage, mean time interval in hours and minutes.

\section{Results}

A total of 30 patients were admitted at the PGH Emergency Room with suspected acute coronary syndrome from September to October of 2011. There were equal numbers of males and females. The age range was 39 to 80 years old with a mean age of 59 years. One third of the patients hailed from Cavite, while 23.3\% came from Manila. Typical angina was the chief complaint of the majority of the patients $(79.3 \%)$.

There were equal numbers of patients with STEMI $(43 \%)$ and NSTEMI (43\%). The rest of the patients $(14 \%)$ were eventually deemed to have other problems other than acute coronary syndromes as the cause of their admission (e.g., gastroesophageal reflux, chronic stable angina). The majority of patients $(83.33 \%)$ had previous episodes of anginal chest pains, most of whom had chest pains for more than 2 weeks prior to their current admission.

The most common risk factor was hypertension (72.4\%) followed by smoking (34.48\%). Around 20\% were diabetics, and $31 \%$ had known lipid abnormalities or were on lipidlowering agents. Around one third of the patients had previous admissions for acute coronary events. Only two patients had angiographically confirmed CAD.

The majority of patients assessed to have acute coronary syndromes (26 patients) were in Killip Class I. Only one was in cardiogenic shock upon admission. The majority of patients were eventually transferred to the Medical ICU (40\%), while a significant proportion (30\%) was sent home after being managed solely at the emergency department (Table 1).
Table 1. Baseline characteristics of ACS patients

\begin{tabular}{|c|c|}
\hline Age, mean & 59 years \\
\hline \multicolumn{2}{|l|}{ Sex } \\
\hline Males & $16(53 \%)$ \\
\hline Females & $14(47 \%)$ \\
\hline \multicolumn{2}{|l|}{ Home address } \\
\hline Cavite & $10(33.3 \%)$ \\
\hline Manila & $7(23.3 \%)$ \\
\hline Laguna & $4(13.3 \%)$ \\
\hline \multicolumn{2}{|l|}{ Chief complaint } \\
\hline Typical angina & $23(79.3 \%)$ \\
\hline Epigastric pain & $2(6.9 \%)$ \\
\hline Dyspnea & $2(6.9 \%)$ \\
\hline \multicolumn{2}{|l|}{ Type of ACS } \\
\hline STEMI & $13(43.3 \%)$ \\
\hline UA/NSTEMI & $13(43.3 \%)$ \\
\hline Unstable Angina & $4 \quad(13.3 \%)$ \\
\hline \multicolumn{2}{|l|}{ Smoking history } \\
\hline Current smoker & $10(34.48 \%)$ \\
\hline Pervious smoker & $5(17.24 \%)$ \\
\hline Nonsmoker & $14(48.28 \%)$ \\
\hline Known diabetes & $6(20.69 \%)$ \\
\hline Known hypertension & $21(72.4 \%)$ \\
\hline Known dyslipidemia & $9(31 \%)$ \\
\hline Previous ACS & $10(34.48 \%)$ \\
\hline \multicolumn{2}{|l|}{ Prior angina } \\
\hline$<2$ weeks & $11(37.43 \%)$ \\
\hline$>2$ weeks & $14(48.28 \%)$ \\
\hline Angiographically confirmed CAD & $2(6.9 \%)$ \\
\hline Previous stroke & $4(13.79 \%)$ \\
\hline \multicolumn{2}{|l|}{ Pre-hospitalization medications } \\
\hline Aspirin & $9(31 \%)$ \\
\hline Clopidogrel & $6(20.69 \%)$ \\
\hline ACE-inhibitors & $7(24.14 \%)$ \\
\hline ARBs & $3(10.34 \%)$ \\
\hline Beta-blockers & $7(24.14 \%)$ \\
\hline Calcium-channel blockers & $2(6.9 \%)$ \\
\hline Lipid-lowering agents & $7(24.14 \%)$ \\
\hline Nitrates & $10(34.48 \%)$ \\
\hline Digoxin & $3(10.34 \%)$ \\
\hline \multicolumn{2}{|l|}{ Killip Class $(n=26)$} \\
\hline Class I & $21(72.4 \%)$ \\
\hline Class II & $4(13.79 \%)$ \\
\hline Class III & 0 \\
\hline Class IV & $1(3.45 \%)$ \\
\hline \multicolumn{2}{|l|}{ Disposition } \\
\hline Medical ICU & $12(40 \%)$ \\
\hline Ward & $7(23.3 \%)$ \\
\hline Home & $9(30 \%)$ \\
\hline Home against advise & $1(3.3 \%)$ \\
\hline
\end{tabular}

Time of symptom onset could not be determined in 5 out of the 30 patients because this was not specified in the clinicians' history. Among those with available data, average time from onset of symptoms to ER consult was 9 hours, 38 minutes. Almost half of these patients (10 patients or $42 \%$ ) consulted between 5 and 8 hours from symptom onset. There were 6 patients who consulted after 12 hours but most of these patients were previously seen at other institutions. There was only one patient without an arrival time recorded in the ER triage logbook. However, time of first physician contact could not be determined in 16 patients (53\%) because of the lack of documentation. Among those with available data, the mean interval between hospital arrival to first 
physician contact was 45 minutes. Door-to-ECG time was obtainable in 28 patients and was found to be almost 2 hours. Half of these patients had their first ECG within 30 minutes to 90 minutes upon arrival at the ER. Six patients (21\%) had their first ECG more than 3 hours after arrival at the ER. There were 6 patients who received thrombolytic therapy. The door-to-decision time was 2 hours, 22 miniutes, while the door-to-needle time was 3 hours, 37 minutes. Half of those who received thrombolytic therapy received the intervention between 4.5 and 5 hours after admission to the ER. The average length of stay of patients in the emergency room before they are transferred or discharged is 13 hours. The most common disposition was the medical ICU $(40 \%$, Table 2).

Table 2. Time intervals to diagnosis and treatment

\begin{tabular}{lll}
\hline \multicolumn{1}{c}{ Time Interval } & \multicolumn{1}{c}{$\begin{array}{c}\text { Study } \\
\text { Results }\end{array}$} & $\begin{array}{c}\text { International } \\
\text { Standards } \\
\text { (ACC/AHA) }\end{array}$ \\
\hline Symptom onset to ED consult, mean $(\mathrm{n}=25)$ & $9 \mathrm{hrs}, 38 \mathrm{mins}$ & 20 minutes \\
Door-to-first-MD time, mean $(\mathrm{n}=13)$ & $45 \mathrm{mins}$ & 20 minutes \\
Door-to-ECG time, mean $(\mathrm{n}=28)$ & $1 \mathrm{hr}, 49$ mins & 10 minutes \\
Door-to-decision time, mean $(\mathrm{n}=6)$ & $2 \mathrm{hrs}, 22$ mins & 30 minutes \\
Door-to-needle time, mean $(\mathrm{n}=6)$ & $3 \mathrm{hrs}, 37$ mins & 30 minutes \\
Length of ER stay, mean $(\mathrm{n}=26)$ & $13.4 \mathrm{hrs}$ & 6 to 12 hours \\
\hline
\end{tabular}

Table 3. Pharmacologic process-of-care indicators

\begin{tabular}{lcc}
\hline \multicolumn{1}{c}{ Pharmacologic Therapies } & $\begin{array}{c}\text { Patients eligible for } \\
\text { Therapies }\end{array}$ & Percentage \\
\hline ASA within 24h $(\mathrm{n}=29)$ & 27 & $93 \%$ \\
Thienopyridines $(\mathrm{n}=28)$ & 27 & $96 \%$ \\
Anticoagulants $(\mathrm{n}=26)$ & 22 & $84 \%$ \\
Statins $(\mathrm{n}=27)$ & 26 & $96 \%$ \\
B-blockers $(\mathrm{n}=22)$ & 20 & $91 \%$ \\
ACE-inhibitors or ARBs $(\mathrm{n}=26)$ & 23 & $88 \%$ \\
Fibrinolytic therapy $(\mathrm{n}=9)$ & 6 & $67 \%$ \\
\hline
\end{tabular}

Twenty-seven out of 29 patients were eligible for aspirin administration. Out of those eligible, 93\% received aspirin. On the other hand, all except one patient among those eligible received thienopyridines. The majority $(84 \%)$ of patients eligible for anticoagulant therapy were given anticoagulants. Fourteen out of these 22 patients received unfractionated heparin while the rest received low molecular heparin. Statin therapy was given to all except one of those who were eligible. There was also a high prevalence of the use of beta-blockers and ACE-inhibitors among those without any contraindications (91\% and $88 \%$, respectively). There were 9 patients out of 13 patients with STEMI who were deemed to be eligible for fibrinolytic therapy. The rest of the patients presented beyond 12 hours of chest pain onset and were already chest-pain free. Among those who were eligible for thrombolysis, $67 \%$ received thrombolytic therapy with streptokinase. No patient was given the thrombolytic agent within 30 minutes upon admission to the emergency department (Table 3). No patient underwent cardiac catheterization as the primary means of revascularization. Hence, door-to-balloon time could not be determined.

\section{Discussion}

The clinical characteristics of patients admitted at the PGH Emergency Room highlights its role as a tertiary referral center. More than half of the patients were residents of communities south of Manila (Paranaque, Las Piñas, and the provinces of Laguna and Cavite). Interestingly, one in every three patients admitted for ACS is from Cavite, outnumbering those who come from the cities of Manila or Pasay. This could be due to the proximity of PGH to Cavite, making the hospital highly accessible to its residents. This could also be due to the paucity of public hospitals in the areas south of Manila, capable of giving adequate emergency management for ACS, which compels patients from these areas to go all the way to PGH to seek medical attention.

In this study, almost half of the patients admitted had ST-elevation MI. This again highlights the role of PGH as a tertiary referral center, where the emergency room triage system prioritizes cases that are deemed to be more critical over those who can be managed in smaller hospitals. This study did not include patients who were redirected to other institutions, which could have increased the number of patients with unstable angina or non-ST elevation MI. Hence, the number of ACS cases consulting at PGH that is reported in this data may be an underestimate.

The majority of patients complained of typical angina. Only a few patients had atypical manifestations such as epigastric pain and dyspnea. Again, in our study, it is not surprising to see that more than a third of the entire population included already had history of previous admission for ACS.

Hypertension is the most prevalent comorbidity among patients admitted for ACS at PGH. In fact, three out of every four patients who were admitted claimed to have a history of elevated blood pressure. This high prevalence of hypertension in this group of patients correlates with the high prevalence of hypertension in the general Filipino population. The 2008 National Nutrition and Health Survey (NNHeS II) showed that 25\% of Filipinos have hypertension defined as a blood pressure reading equal to or higher than $140 / 90 \mathrm{mmHg} .{ }^{10}$ Half of the patients included in this study were either current smokers or previous smokers. This also correlates with the NNHeS II data which shows a high prevalence of smoking in the general Filipino population (34\%).

One third of the patients included in this study claimed to be dyslipidemic or taking a lipid-lowering agent. This may be influenced by the high percentage of patients who have had previous admissions for acute coronary syndromes in this population. This also reflects the high prevalence of 
dyslipidemia in the general population, which, according the NNHeS II, is at a staggering $64 \%$ for low HDL and $10 \%$ for elevated total cholesterol levels.

In this study, one of every five patients admitted for ACS has a history of diabetes. The NNHeS II data showed that one in every 20 (4.8\%) of Filipinos have elevated fasting blood sugar (FBS) and this prevalence of high FBS peaks at age 50-59 years old. A patient admitted at the PGH Emergency Room is initially seen by the triage physician who will get a short history and a targeted physical examination. Every patient who consults at the ER will be recorded in the triage logbook, including the time of consult and the triage disposition. If the ER is congested and beyond its capacity, patients who are stable and can still be transferred to other institutions are advised to transfer. However, if the triage physician decides that a particular patient needs emergent care, the patient will then be decked to a treatment officer-an ER medicine resident or an internal medicine resident who will manage the patient accordingly.

In this study, we demonstrated that the mean time from symptom onset to arrival at the PGH-ER, as reflected by the triage logbook, is 9 hours and 30 minutes. This, however, did not take into account whether or not the patient received earlier care at another facility. Prior admission at another facility will increase the measured lag time between symptom onset and PGH-ER arrival. Also, data was not available in a substantial number $(17 \%)$ of the patients due to lack of documentation of time of symptom onset or time of ER arrival.

The treatment officer is the first physician to provide initial management to patients at the ER. In this study, more than half of the physicians failed to document the time of their first contact with the patient. This underscores the need for appropriate and sufficient documentation of critical time intervals for adequate quality of care improvements to be set in place. Among those with obtainable data, this study shows that patients wait for 45 minutes before the initial orders for management are made.

As was recommended by guidelines from major international organizations, the Clinical Practice Guidelines for the Management of Coronary Artery Disease made by the Philippine Heart Association in 2009 recommend that an electrocardiogram must be obtained within 10 minutes of admission for all patients suspected to have ACS. Our study shows that the mean door-to-ECG time at the PGH ER is 1 hour, 49 minutes. This is 10 times longer than the recommended door-to-ECG time. This finding reflects gaps in the health care delivery systems in the hospital. The absence of a dedicated machine in the emergency room has already been cited by a previous study by Magno et al. in $2010 .{ }^{11}$ In that study, the mean door-to-ECG time was similar to our study (109 minutes). One year later, despite recommendations to provide a dedicated ECG machine at the ER, the door-to-ECG time remains remarkably high. Our study, however, did not assess whether or not this delay could affect patient outcome.

The decision to thrombolyse patients with STEMI relies heavily on the cardiology fellow on duty. Upon seeing the ECG, the treatment officer must immediately notify the cardiology service. For free streptokinase to be released by the pharmacy, the requisition voucher must have the signature of the cardiology fellow. After all the required papers are completed, streptokinase must be obtained from the central block pharmacy before it can be administered by the ER nurses to the patients.

In this study, only 9 out of 13 patients were eligible for thrombolysis. The other patients have already exceeded the "golden period" for thrombolytic therapy. Only 6 of these eligible patients were given the medication. Furthermore, it required more than 2 hours after patient arrival before a decision to thrombolyse was made. Another hour was needed before thrombolysis could actually be initiated. Hence, the door-to-needle time in our study was seven times longer than the guideline recommended target door-toneedle time of 30 minutes.

As could be expected in a charity hospital setting, no patient underwent primary percutaneous intervention despite the availability of a cardiac catheterization laboratory in the institution. The lack of finances is the most obvious reason for this. This underscores the importance of thrombolytic therapy as the most practical and readily available revascularization modality in the institution. Hence, its use must be maximized.

In our study, compliance to guideline-recommended emergency room medications is very high. The appropriateness of these medications for individual patients, however, was not assessed. Also, the timing of medication delivery was not determined in this study due to the lack of documentation by physicians and/or nursing staff regarding the timing of medication delivery.

\section{Conclusion}

In this study, there were equal numbers of STEMI patients and NSTEMI/UA patients at the PGH Emergency Room from September to October 2011. There were equal numbers of males and females with a mean age of 59 years. The most common comorbidities were hypertension, followed by smoking and dyslipidemia. The door-to-ECGtime and door-to-needle time remain remarkably above the guideline-recommended time targets, with a door-to-ECG time of almost 2 hours, and a door-to-needle time of more than 3.5 hours. The compliance for class I medications among patients admitted for ACS from September to October 2011 was optimal. 


\section{Limitations of the Study}

The study relied heavily on notifications of physicians at the ER and the investigators' regular inquiry of current ACS patients for inclusion of subjects into the registry. Because no patient contact between the investigators and the patients took place, information obtained relied heavily on a review of medical records. Because this study only lasted for 2 months, the results rely heavily on the speed and competence of the personnel assigned at the ER during that period. A bigger, more exhaustive, 1-year registry is currently underway.

\section{Recommendations}

1. A standardized chest pain protocol in the treatment of acute coronary syndromes at the ER should be evaluated and institutionalized.

2. Timing of patient contact, performance of critical laboratory procedures, and actual administration of medications should be documented. All medical personnel involved in these must be mandated to do so.

3. A clinical registry that will regularly evaluate the delivery of health services using specific quality performance indicators must be set in place. This registry should be actively participated in by all services involved in the ER management of ACS patients.

4. A nationwide, multi-institution registry that would not only examine the clinical profile of patients but also assess the quality of health care delivery must be set in place. A standardized emergency room data form will help address gaps in information required.

\section{References}

1. Department of Health. The 2005 Philippine Health Statistics [Online]. 2005 [cited 9 September 2010]. Available from http://www.doh.gov.ph/files/phs2005.pdf.

2. Libby P, Bonow R, et al. Braunwald's Heart Disease: Textbook of Cardiovascular Medicine, 8th ed. Philadelphia: Elsevier; 2007. pp.1220 1222.

3. Philippine Heart Association. Clinical Practice Guidelines for the Management of Coronary Artery Disease. July 2009.

4. Solomon DJ, Henry RC, Hogan JG, et al. Evaluation and implementation of public health registries. Pub Health Rep. 1991; 106(2):142-50.

5. Roe MT, Messenger JC, Weintraub WS, et al. Treatments, trends, and outcomes of acute myocardial infarction and percutaneous coronary intervention. J Am Coll Cardiol. 2010; 56(4):254-63.

6. GRACE ACS Risk Model: Welcome to COR, JAMA [Online]. June 9, 2004 BMJ [cited 9 September 2010]. Available from http://www.outcomes-umassmed.org/GRACE/index.

7. Goodman SG, Huang W, Yan AT, et al. The Expanded Global Registry of Acute Coronary Events: Baseline characteristics, management practices, and hospital outcomes of patients with acute coronary syndromes. Am Heart J. 2009; 158(2):193-201.

8. Peterson ED, Shah BR, Parsons L, et al. Trends in quality care for patients with acute myocardial infarction in the National Registry of Myocardial Infarction from 1990 to 2006. Am Heart J. 2008; 156(6):104555 .
9. Obrado M, Punzalan FE. Practice Pattern of Physicians in the In-hospital Management of Acute Coronary Syndrome and their Compliance to Existing Guidelines: A Quality of Care Study. UP-PGH Cardiovascular Section, 2008. Unpublished.

10. Department of Science \& Technology Philippines. 7th National Nutrition Survey [Online]. 2008. [cited 30 March 2011]. Available from www.fnri.dost.gov.ph/

11. Magno JD, Santella C, Punzalan FE. Timely Rhythm Assessment for Chest Pain at the Emergency Room (TRACER). UP-PGH Cardiovascular Section, 2010. Unpublished. 


\section{Appendix}

Appendix 1. Data collection form

\section{Acute Coronary Events at the Emergency Room: The Philippine General Hospital Registry (ACER-PGH)}

PART 1. *Write "N/A" for data not available in patient's chart. Study ID :

Data taken by:

\section{PATIENT DATA: (to be filled up by ER resident)}

Patient's Initials:

Age:

PGH Case \#:

Home address:

Sex: [ ] Male [ ] Female

Place/address where chest pain occurred:

Chest pain onset: Date:

Date of arrival at PGH-ER:

Time:

Time of arrival at PGH-ER:

[Date of umival as rolectod in ER riago logbook]

[Timo of arivel as roloctod in ER triags logbook]

IIMIE-IN-MOTION: (to be filled up by investigator)

Time seen by ER-Treatment Officer:

Time admitted to PGH ER:

[Timo intial doctor's osders waso made]

Time of first ECG: [Time rellectod in czse recosd]

If applicable,

Time seen by IM resident: Timo of Sist IM rosideat's osery in chast]

Time seen by Cardio fellow: [Timos of Cudio Eollow's entry in char:]

For STEMII patients,

Time of decision for reperfusion:

Time of administration of fibrinolytic agent: Time of first balloon inflation, if applicable:

[Time orders for teperfusion ware writtes]

[Time fibrinolytic wes startad]

\section{CARDIAC ENZYMES:}

\begin{tabular}{|l|l|l|l|}
\hline & Time Ordered & Time Processed & Time Noted by MD \\
\hline CK-MB & & & \\
\hline Troponin I & & & \\
\hline Troponin T & & & \\
\hline
\end{tabular}

ER MEDICATIONS:

\begin{tabular}{|l|l|l|}
\hline & Time ordered by MD & $\begin{array}{l}\text { Time given [Time vinsos } \\
\text { camed out MD's ordser] }\end{array}$ \\
\hline Aspirin & & \\
\hline Thienopyridine & & \\
\hline UFH/LMWH & & \\
\hline Beta-blockers & & \\
\hline Statins & & \\
\hline ACE-inhibitor/ ARB & & \\
\hline Nitrates & & \\
\hline Others: & & \\
\hline & & \\
\hline
\end{tabular}

DISPOSIIION: [ ] Senthome [ ] Ward [ ] ICU [ ] Expired [ ] HAMA

Date disposed:

Time disposed:

[Date Time received in ICU or ward if admitted, of resident's discharge orders if sent home, or time of death in death certificate if expired] 


\section{PART 2. Patient Characteristics}

Patient' Initials:

PGH Case Number:

Study ID :

I. Baseline Characteristics

$\begin{array}{rlr}\text { Prior angina } & \text { Yes ( ) No Nonown ( ) } \\ \text { if yes, indicate if present: } \quad[0<2 \text { weeks before }\end{array}$

Prior MI Yes ( ) No ( ) Unknown ( )

$\begin{array}{llll}\text { Prior CHF } & \text { Yes ( ) } & \text { No ( ) inknown ( ) } & \\ \text { PCI } & \text { Yes ( ) } & \text { No ( ) if yes, indicate year: } & \text { Unknown ( ) } \\ \text { Previous CABG } & \text { Yes ( ) } & \text { No ( ) if yes, indicate year: } & \text { Unknown ( ) }\end{array}$

Prior catheterization with stenosis $>\mathbf{5 0} \%$

Yes ( ) No ( ) if yes, indicate year:___ Unknown ( )

History of stroke Yes ( ) No ( ) if yes, indicate year of most recent:___ Unknown ( )

If yes, type of stroke: ( ) Hemorrhagic (subdural or subarachnoid) ( ) Non-Hemorrhagic ( ) Unknown

History of TIA $\quad$ Yes ( ) No( ) Unknown ( )

PAD $\quad$ Yes ( ) No ( ) Unknown ( )

Carotid Artery Disease Yes ( ) No ( ) Unknown ( )

Diabetes Yes ( ) No( ) Unknown ( )

Type of Diabetic Control (check all that apply)

No treatment:

Diet:

Yes ( ) No( )

Oralion OHA:

On Insulin treatment:

Insulin alone:

Yes ( )

No( )

Yes ( ) No( )

Yes ( ) No( )

Yes ( ) No( )

Hypertension Yes ( ) No( ) Unknown ( )

Smoking History

Current: $\quad$ Yes ( ) No( )

Former: $\quad$ Yes ( ) No( )

Never smoked: Yes ( ) No( )

Dyslipidemia
Family History of CAD ( ) Yes ( ) No( ) No( ) Unknown ( )

Chronic lung disease Yes ( ) No( ) Unknown ( )

Chronic kidney disease $\quad$ Yes ( ) No( ) Unknown( )

II. Pre hospital Medications

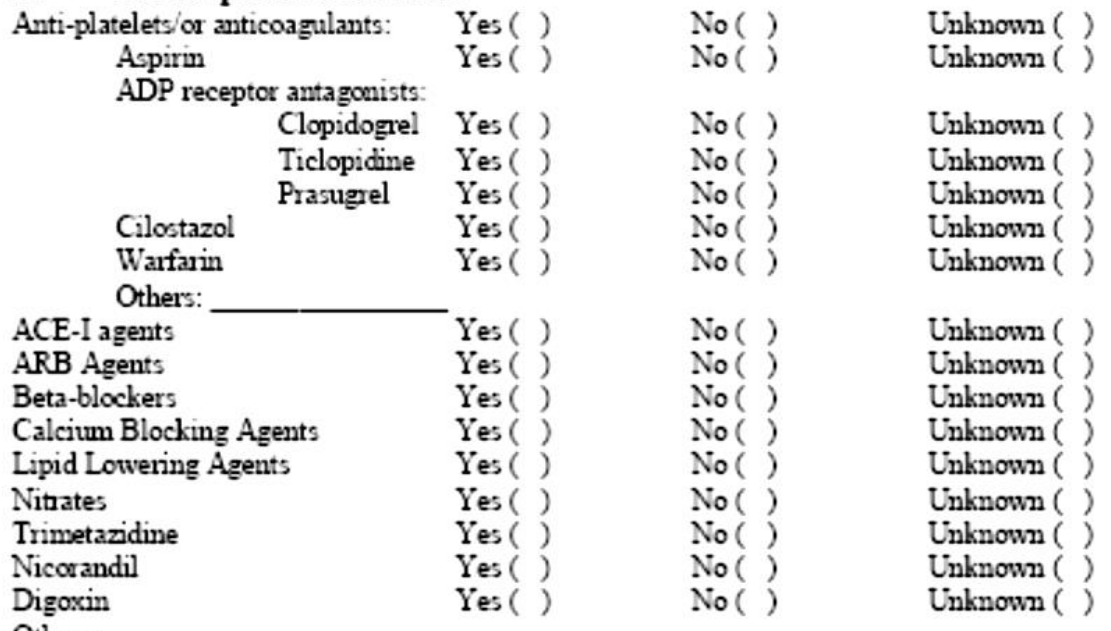

Others: 


\section{Clinical Presentation}

ACS predominant symptom:

( ) Chest pain or pressure or heaviness

( ) Atypical/Anginal Equivalents (check all that applies)
( ) arm or jaw pain
( ) nausea/vomiting
( ) dyspnea
( ) choking sensation
( ) fatigue
( ) syncope.
( ) epigastric pain

( ) Cardiac arrest/aborted sudden death

( ) Others:

Means of transport

( ) Self family

( ) Ambulance

( ) Mobile ICU

( ) Air transfer from another facility

( ) Ambulance transfer from another acute care facility

( ) Others:

Killip Class of patient at time of admission
( ) I
( ) II
( ) III
( ) IV

\section{Vital Signs at the time of presentation:}

Blood pressure: SBP: -

Heart rate (bpm):

Height $(\mathrm{cm})$ :____ Weight $(\mathrm{kg})$ :

BMI:

IV. Laboratory Tests:

12-LECG Date:___ Time:

( ) ST-Elevation Leads:

( ) Left-bundle-branch block

( ) ST-segment depression Leads:

( ) T-wave inversion Leads:

( ) Others:

Cardiac Enzymes:

CKMB

Date: Time:

CK-Total

Troponin I

Troponin T

Total serum cholesterol $(m \bar{g} / \mathrm{dl})$

LDL

HDL

Triglycerides

Serum creatinine (umol/L)

FBS (mg/dL)

CBC:

\section{$\mathrm{Hb}(\mathrm{g} / \mathrm{dl})$}

Hct $(\%)$

WBC diff count

Chest X-ray

Platelet Count
( ) Cardiomegaly
( ) Congestion
( ) Others: 


\section{Appendix 2. Definition of terms}

1. Acute coronary syndromes (ACS)

- or acute coronary events (ACE); a spectrum of clinical conditions ranging from unstable angina to non-ST-elevation myocardial infarction (NSTEMI) to ST-elevation myocardial infarction (STEMI)

2. Unstable angina (UA)

- $\quad$ Final diagnosis of ACS but with negative cardiac enzyme findings

3. Non-ST Elevation Myocardial Infarction (NSTEMI)

- ACS with no new ST-elevation seen on index or on any subsequent ECG, with one or more positive cardiac enzymes based on ranges used by the processing laboratory

4. ST-elevation Myocardial Infarction (STEMI)

- a history suggestive of acute myocardial infarction associated with at least $0.1 \mathrm{mV}$ ST segment elevation on at least

2 contiguous leads on the ECG or new or presumed new left-bundle branch block

5. Quality indicator

- a process of care measure that is linked to improved patient outcomes by clinical trials, can be measured through existing data sources, and is objectively quantifiable. This is expressed as a ratio with the denominator defined as all patients eligible to receive care and the numerator as all patients who actually received care

6. Door-to-Data Time

- time from the arrival of the patient to the emergency department, as reflected in the Department of Emergency Medicine physician's triage logbook to the time a 12-L ECG is done, as written by the ECG technician on the ECG tracing

7. Data-to-Decision Time

- time from the conduct of the actual ECG to the time a decision for reperfusion therapy was made, as reflected by the time the attending physician wrote the orders for reperfusion in the patient's chart

8. Decision-to-Needle Time

- time from when the orders for reperfusion were given to the actual administration of the fibrinolytic agent, as reflected in the nurses' notes

9. Decision-to-Balloon Time

- time from when the orders for primary percutaneous coronary intervention were made, as reflected in the chart entry, to the time of the first balloon inflation

10. Cardiogenic shock

- the presence of any or more of the following: systolic blood pressure $<90 \mathrm{mmHg}$ for at least 30 minutes, or the need for supportive measures to maintain a systolic blood pressure $>90 \mathrm{mmHg}$, or end-organ hypoperfusion (cool extremities or urine output $<30 \mathrm{ml} /$ hour and a heart beat $>60 \mathrm{bpm}$ ), or hemodynamic criteria of $\mathrm{CI}<2.2 \mathrm{~L} / \mathrm{min}$ per square meter body surface area and a pulmonary capillary wedge pressure of at least $15 \mathrm{mmHg}$

11. Recurrent ischemia

- the presence of ischemic type of discomfort at rest and lasting at least 10 minutes, and is associated with new, horizontal, or downsloping ST segment deviation (elevation $>0.1 \mathrm{mV}$ or depression $>0.05 \mathrm{mV}$ measured $80 \mathrm{msec}$ after the J-point in $>2$ contiguous precordial leads or $>2$ adjacent limb leads)

12. Failed thrombolysis

- the persistence of chest pain, cardiogenic shock, or failure of the ST elevation to decrease by $50 \%$, at least 90 minutes since the administration of fibrinolytic therapy 


\section{Appendix 3. Eligibility criteria for specific therapies}

\section{Eligibility for Fibrinolytic Therapy}

Patients are eligible for fibrinolytic therapy if they present to the emergency room $<12$ hours after the onset of symptoms and if they have no contraindication to fibrinolysis which include the following:

Any prior intracranial hemorrhage

Known structural cerebral vascular lesion (e.g. arterio-venous malformation)

Known malignant intracranial neoplasm (primary or metastatic)

Ischemic stroke within 3 months except acute ischemic stroke within 3 hours

Suspected aortic dissection

Active bleeding or bleeding diathesis (excluding menses)

Significant closed head or facial trauma within 3 months

\section{Eligibility for Beta-Blocker Therapy}

Heart rate not persistently below 50 beats/minute

Systolic blood pressure not persistently less than $100 \mathrm{~mm} \mathrm{Hg}$

Absence of $2^{\text {nd }}$ or $3^{\text {rd }}$ degree atrioventricular block

Absence of bronchospasm

\section{Eligibility for Aspirin or Clopidogrel Therapy}

No history of aspirin or clopidogrel allergy

No history of gastrointestinal bleeding or ulcer

\section{Eligibility for ACE-inhibitors}

No hyperkalemia

No severe renal failure (creatinine clearance $<30 \mathrm{~mL} / \mathrm{min}$ )

\section{Eligibility for statins}

No history of previous intolerance

No active liver disease 